\title{
USO DOS ÓLEOS ESSENCIAIS EM ONICOMICOSE: REVISÃO INTEGRATIVA
}

\author{
Taynnar Barbosa Ribeiro' (D), Jefferson Abraão Caetano Lira1,3 (D), Sandra Marina Gonçalves

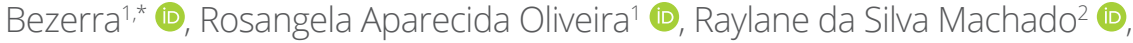 \\ Lídya Tolstenko Nogueira3 ${ }^{\text {(D) }}$
}

\section{RESUMO}

Objetivo: identificar na literatura como os óleos essenciais são utilizados em onicomicose. Métodos: revisão integrativa com buscas realizadas nas bases de dados MEDLINE/PubMed, Web of Science, Cochrane, SCOPUS e BVS, sendo 13 estudos analisados. Resultados: o uso de óleos essenciais Melaleuca alternifólia, Lavandula angustifólia, Eucalyptus citriodora e Foeniculum vulgare mostram-se eficazes e trazem benefícios no tratamento da onicomicose, por inibirem o crescimento micelial dos fungos infectantes das unhas. Sendo assim, os profissionais de saúde envolvidos nos cuidados em pessoas com onicomicose devem se manter atualizados sobre tais alternativas de tratamento e consequentemente da melhora da assistência prestada a esses pacientes. Conclusão: a eficácia antifúngica de uma variedade de óleos foi comprovada com diferentes formas de apresentação e possíveis associações. Entretanto, o nível da evidência apresentado foi baixo, o que justifica a necessidade de estudos mais robustos sobre a temática.

DESCRITORES: Estomaterapia. Onicomicose. Doenças da unha. Óleos voláteis.

\section{USE OF ESSENTIAL OILS IN ONYCHOMYCOSIS: AN INTEGRATIVE REVIEW}

\begin{abstract}
Objective: identify in the literature how essential oils are used in onychomycosis. Methods: integrative review with searches performed in the MEDLINE/PubMed, Web of Science, Cochrane, SCOPUS and BVS databases, with 13 studies analyzed. Results: the use of essential oils Melaleuca alternifolia, Lavandula angustifolia, Eucalyptus citriodora and Foeniculum vulgare are effective and bring benefits in the treatment of onychomycosis, as they inhibit the mycelial growth of the nail fungus. Therefore, health professionals involved in the care of people with onychomycosis must keep themselves updated on such treatment alternatives and, consequently, on improving the care provided to these patients. Conclusion: the antifungal efficacy of a variety of oils has been proven with different forms of presentation and possible associations. However, the level of evidence presented was low which justifies the need for more robust studies on the subject.
\end{abstract}

DESCRIPTORS: Stomatherapy. Onychomycosis. Nail diseases. Oils. Volatile.

1. Universidade Estadual do Piauí - Centro de Ciencias da Saúde - Especialização em Estomaterapia - Teresina (PI), Brasil.

2. Universidade Federal do Piauí - (PI), Brasil.

3. Universidade Federal do Piauí - Departamento de Enfermagem - Programa de Pós- Graduação em Enfermagem - Teresina (PI), Brasil.

*Autora correspondente: sandramarina@ccs.uespi.br

Editor de Seção: Juliano Teixeira Moraes

Recebido: Jan. 13, 2021 | Aceito: Abr. 19, 2021

Como citar: Ribeiro TB; Lira JAC; Oliveira RA; Bezerra SMG; Machado RS; Nogueira LT. Uso dos óleos essenciais em onicomicose: revisão integrativa. ESTIMA, Braz. J. Enterostomal Ther., 2021, 19: e1321. https://doi.org/10.30886/estima.v19.1011 PT 


\title{
USO DE ACEITES ESENCIALES EN ONICOMICOSIS: REVISIÓN INTEGRATIVA
}

\begin{abstract}
RESUMEN
Objetivo: Identificar en la literatura cómo se utilizan los aceites esenciales en la onicomicosis. Métodos: Revisión integrativa, con búsquedas realizadas en las bases de datos MEDLINE / PubMed Web of Science, Cochrane, SCOPUS y VHL, donde se analizaron 13 estudios. Resultados: El uso de los aceites esenciales Melaleuca alternifolia, Lavandula angustifolia, Eucalyptus citriodora y Foeniculum vulgare resulta eficaz y aporta beneficios en el tratamiento de la onicomicosis ya que inhiben el crecimiento micelial del hongo ungueal. Así, los profesionales sanitarios implicados en la atención de las personas con onicomicosis onicomicosis deben mantenerse actualizados sobre dichas alternativas de tratamiento y, en consecuencia, sobre la mejora de la atención que se brinda a estos pacientes. Conclusión: La eficacia antifúngica de una variedad de aceites ha sido probada con diferentes formas de presentación y posibles asociaciones. Sin embargo, el nivel de evidencia presentado fue bajo, lo que justifica la necesidad de estudios más robustos sobre el tema.
\end{abstract}

DESCRIPTORES: Estomaterapia Onicomicosis. Enfermedades de la uña. Aceites Volátiles. Aceites esenciales.

\section{INTRODUÇÃO}

Onicomicose é uma infecção fúngica que ocorre nas unhas e pode acometer a pele adjacente. Afeta mais frequentemente as unhas dos pés do que as unhas das mãos e é caracterizada por descoloração da unha, espessamento da placa ungueal e onicólise. É a patologia ungueal mais comum e representa cerca de $90 \%$ das infecções de unha em todo o mundo ${ }^{1}$.

A prevalência da doença aumenta com uma variedade de fatores de risco, como idade avançada, morfologia anormal das unhas, imunodeficiência e fatores genéticos. A onicomicose constitui um problema de saúde substancial, pois pode ter consequências negativas para a saúde, como dor, desconforto e comprometimento físico ${ }^{2}$.

Estudos nacionais e internacionais trazem os dermatófitos como os agentes etiológicos mais encontrados nas onicomicoses, tendo representação pelos gêneros Trichophyton, Microsporum e Epidermophyton, seguidos pelas leveduras, representadas em sua maior parte pelo gênero Candida, assim como pelos fungos filamentosos não dermatófitos, em que os Scopulariopsis, Fusarium e Aspergillus são os principais gêneros ${ }^{3-5}$.

Em países com maior desenvolvimento, o uso de calçados fechados, característico dos grandes centros urbanos, cria um ambiente propenso à onicomicose por fungos filamentosos. A umidade e a alta temperatura no interior do calçado são condições que favorecem o desenvolvimento dos dermatófitos. Por outro lado, andar com os pés descalços em contato com o solo e vegetação é um hábito que favoreceria a onicomicose por fungos não dermatófitos (FND) ${ }^{6,7}$. Diante da apresentação da patologia e sua complexidade, destaca-se o uso de óleos essenciais em pacientes com onicomicose, a fim de atuar de forma benéfica no seu controle.

Os óleos essenciais são compostos orgânicos muito perfumados e voláteis obtidos através da extração de várias partes das plantas. Geralmente tem consistência aquosa e límpida, porém podem ficar sólidos em temperaturas mais baixas. São solúveis em álcool, éter e outros compostos graxos; insolúveis em água e podem não ter cor ou apresentar desde tons claros até mais escuros e opacos. O Brasil ocupa uma posição reconhecida na produção de óleos essenciais, ao lado da Índia, China e Indonésia, que estão na lista dos quatro maiores produtores mundiais ${ }^{8}$.

Os óleos essenciais são denominados voláteis, pois quando estão em contato com o ar, em temperatura ambiente, evaporam. Refringentes ou etéreos são outras denominações que podem ser encontradas. Porém, o termo mais utilizado é óleo essencial, pois esses representam as "essências" ou compostos odoríferos das plantas". Os terpenos, ésteres, éteres, álcoois, fenóis, aldeídos, óxidos, cetonas, ácidos orgânicos e demais componentes detectados como elementos traços são os principais constituintes ${ }^{10}$. 
Ao falar da ação fisiológica e/ou farmacológica dos óleos essenciais, o efeito é parecido com de um medicamento ou cosmético, ou seja, de acordo com as substâncias químicas encontradas no óleo. Essas ações poderão ser: analgésica, antibiótica, anti-inflamatória, antisséptica, antitóxica, adstringente, bactericida, depurativa, diurética, desinfetante, solvente, estimulante, entre outras ${ }^{11}$.

Dessa forma, a realização deste estudo sobre uso de óleos essenciais em onicomicose visa buscar dados relevantes sobre a temática ainda pouco estudada, mostrando, assim, a importância do desenvolvimento de pesquisas clínicas que sirvam de subsídio para prevenção e para uso da equipe multiprofissional com ênfase no cuidado especializado pela Estomaterapia. Os óleos essenciais são considerados materiais de fácil aplicabilidade e acesso, características que podem proporcionar um cuidado diferenciado frente às necessidades desse público. Nesse contexto, esta revisão objetiva identificar na literatura como os óleos essenciais são utilizados em onicomicose.

\section{MÉTODO}

Revisão integrativa da literatura, guiada em seis fases: definição da questão de pesquisa, amostragem ou busca na literatura, extração dos dados dos estudos incluídos, avaliação das produções, interpretação dos resultados e síntese do conhecimento ou apresentação da revisão ${ }^{12}$. Para a elaboração da questão norteadora, utilizou-se a estratégia PICo, definindo-se: $\mathrm{P}$ = população: "estudos originais", $\mathrm{I}$ = interesse: "óleos essenciais" e Co = contexto: "onicomicose". Portanto, a questão deste estudo foi: como os óleos essenciais são utilizados em onicomicose?

Tiveram inclusão os estudos de fonte primária, sem delimitação temporária, que tratassem do uso de óleos essenciais no tratamento da onicomicose. Definiram-se como critérios de exclusão estudos publicados apenas como resumo, teses, dissertações e editoriais. A busca ocorreu entre os meses de julho e agosto de 2019, mediante consulta nas seguintes bases de dados: MEDLINE/PubMed (Medical Literature Analysis and Retrievel System Online), Cochrane, Web of Science, SCOPUS e BVS (Biblioteca Virtual em Saúde). Ressalta-se que os artigos duplicados em mais de uma base de dados foram contabilizados apenas uma vez.

A seleção dos descritores foi feita por meio de consulta aos termos dos Descritores em Ciências da Saúde (DeCS) para base de dados BVS e Medical Subject Headings (MeSH) para as demais bases. Observando as peculiaridades e características distintas de cada base de dados, foram construídas estratégias sistemáticas utilizando a busca avançada. Os descritores controlados e não controlados, dentro de cada conjunto de termos da estratégia PICo, foram combinados entre si com o operador booleano OR e, em seguida, cruzados com o operador booleano AND, conforme apresentado na Tabela 1.

Inicialmente, foram encontradas 58 produções, das quais 20 foram removidas do estudo por duplicidade. Após a análise de títulos e resumos, 11 foram excluídos por não serem artigos primários e 4 apresentavam apenas o resumo disponível na base. A leitura de texto completo para avaliar elegibilidade excluiu outros 11 artigos por não responderem à questão de pesquisa, sendo a amostra final composta por 13 artigos. A Fig. 1 descreve o percurso realizado para a identificação, inclusão e exclusão dos estudos, segundo a base consultada.

A extração dos dados foi realizada com auxílio de instrumento próprio, contendo as seguintes informações: autor principal, ano de publicação, título, local e tipo do estudo, objetivo, intervenção, principais resultados e nível de evidência (NE). Para a análise do NE, adotaram-se os conceitos propostos por Melnyk e Fineout-Overholt ${ }^{13}$ : nível I - evidências oriundas de revisão sistemática ou metanálise de todos os ensaios clínicos relevantes randomizados controlados ou provenientes de diretrizes clínicas baseadas em revisões sistemáticas de ensaios clínicos randomizados controlados; nível II - evidências derivadas de pelo menos um ensaio clínico randomizado controlado bem delineado; nível III - evidências obtidas de ensaios clínicos bem delineados sem randomização; nível IV - evidências provenientes de estudos de coorte e de caso-controle bem delineados; nível V - evidências originárias de revisão sistemática de estudos descritivos e qualitativos; nível VI - evidências derivadas de um único estudo descritivo ou qualitativo; e nível VII - evidências oriundas de opinião de autoridades e/ou relatórios de comitês de especialistas. 
Tabela 1. Descritores controlados, não controlados e expressões de buscas utilizadas para recuperação dos artigos. Teresina (PI) - 2019.

\section{$\mathrm{MeSH}$}

\begin{tabular}{|c|c|c|}
\hline \multirow{2}{*}{$\mathbf{P}$} & DC & - \\
\hline & DNC & - \\
\hline \multirow{2}{*}{ I } & DC & "Oils, Volatile", Melaleuca, Clove Oil, Thymus Plant \\
\hline & DNC & Oils, Volatile, Volatile Oils, “Oils, Essential”, Essential Oils, Melaleuca, Clove Oil, Thymus Plant \\
\hline \multirow{2}{*}{$\mathrm{CO}$} & DC & Nail Diseases, Onychomycosis \\
\hline & DNC & Nail Diseases, Nail Disease, Onychomycosis, Onychomycoses, Nail Fungus, Tinea Unguium \\
\hline & SCOPUS & $\begin{array}{l}\text { ( ( TITLE-ABS-KEY ( "Oils, volatile" ) OR TITLE-ABS-KEY ( "volatile oils" ) OR TITLE-ABS-KEY ( "essential } \\
\text { oils" ) OR TITLE-ABS-KEY ( melaleuca ) OR TITLE-ABS-KEY ( "Clove oil" ) OR TITLE-ABS-KEY ("thymus } \\
\text { plant" ) ) AND ( ( TITLE-ABS-KEY ( "nail diseases" ) OR TITLE-ABS-KEY ( "nail disease" ) OR TITLE-ABS- } \\
\text { KEY ( onychomycosis ) OR TITLE-ABS-KEY ( onychomycoses ) OR TITLE-ABS-KEY ( "nail fungus") OR } \\
\text { TITLE-ABS-KEY ( "tinea unguium" )) ) }\end{array}$ \\
\hline
\end{tabular}

\begin{tabular}{|c|c|}
\hline WEB OF SCIENCE & $\begin{array}{l}\text { (TS=("oils, volatile") OR TS=("volatile oils") OR TS=("essential oils") OR TS=(melaleuca) OR TS=("clove oil") } \\
\text { OR TS=("thymus plant")) AND (TS=("nail diseases") OR TS=("nail disease") OR TS=(onychomycosis) OR } \\
\text { TS=(onychomycoses) OR TS=("nail fungus") OR TS=("tinea unguium")) }\end{array}$ \\
\hline $\begin{array}{l}\text { MEDLINE/ } \\
\text { PUBMED }\end{array}$ & $\begin{array}{l}\text { (((((((((("oils, volatile"[MeSH Terms]) OR ("oils, volatile")) OR ("volatile oils"[All Fields])) OR ("essential } \\
\text { oils"[All Fields])) OR ("melaleuca"[MeSH Terms])) OR ("melaleuca"[All Fields])) OR ("clove oil"[MeSH } \\
\text { Terms])) OR ("clove oil"[All Fields])) OR ("thymus plant"[MeSH Terms])) OR ("thymus plant"[All Fields])) } \\
\text { AND (((((((("nail diseases"[MeSH Terms]) OR ("nail diseases"[All Fields])) OR ("nail disease"[All Fields])) } \\
\text { OR ("onychomycosis"[MeSH Terms])) OR ("onychomycosis"[All Fields])) OR ("onychomycoses"[All } \\
\text { Fields])) OR ("nail fungus"[All Fields])) OR ("tinea unguium"[All Fields])) }\end{array}$ \\
\hline
\end{tabular}

COCRHANE

(("oils, volatile") OR ("essential oils") OR (Melaleuca) OR ("clove oil") OR ("thymus plant")) AND (("nail diseases") OR ("nail disease") OR (onychomycosis) OR (onychomycoses) OR ("nail fungus"))

\begin{tabular}{|c|c|c|}
\hline \multicolumn{3}{|r|}{ DeCS } \\
\hline \multirow{2}{*}{$\mathrm{P}$} & DC & - \\
\hline & DNC & - \\
\hline \multirow[b]{2}{*}{ I } & DC & Melaleuca, Óleo de Melaleuca, Óleo de Cravo, Thymus (Planta) \\
\hline & DNC & $\begin{array}{l}\text { Óleos Essenciais, Melaleuca, Óleo de Melaleuca, Óleo de Cravo, Thymus (Planta) Thymus vulgaris, } \\
\text { Tomilho }\end{array}$ \\
\hline \multirow{2}{*}{$\mathrm{CO}$} & DC & Doenças da Unha, Onicomicose \\
\hline & DNC & Doenças da Unha, Onicopatias, Onicomicose, Fungo de Unha, Micose Ungueal, Micose de Unha \\
\hline  & BVS & $\begin{array}{l}\text { (("Óleos Essenciais") OR (mh:(Melaleuca)) OR (Melaleuca) OR (mh:("Óleo de Melaleuca")) OR ("Óleo de } \\
\text { Melaleuca") OR (mh:("Óleo de Cravo")) OR ("Óleo de Cravo") OR (mh:("Thymus (Planta)")) OR ("Thymus } \\
\text { (Planta)") OR ("Thymus vulgaris") OR (Tomilho)) AND ((mh:("Doenças da Unha")) OR ("Doenças da } \\
\text { Unha") OR (Onicopatias) OR (mh:(Onicomicose)) OR (Onicomicose) OR ("Fungo de Unha") OR ("Micose } \\
\text { Ungueal") OR ("Micose de Unha")) }\end{array}$ \\
\hline
\end{tabular}

$D C=$ descritor controlado; DNC = descritor não controlado. 


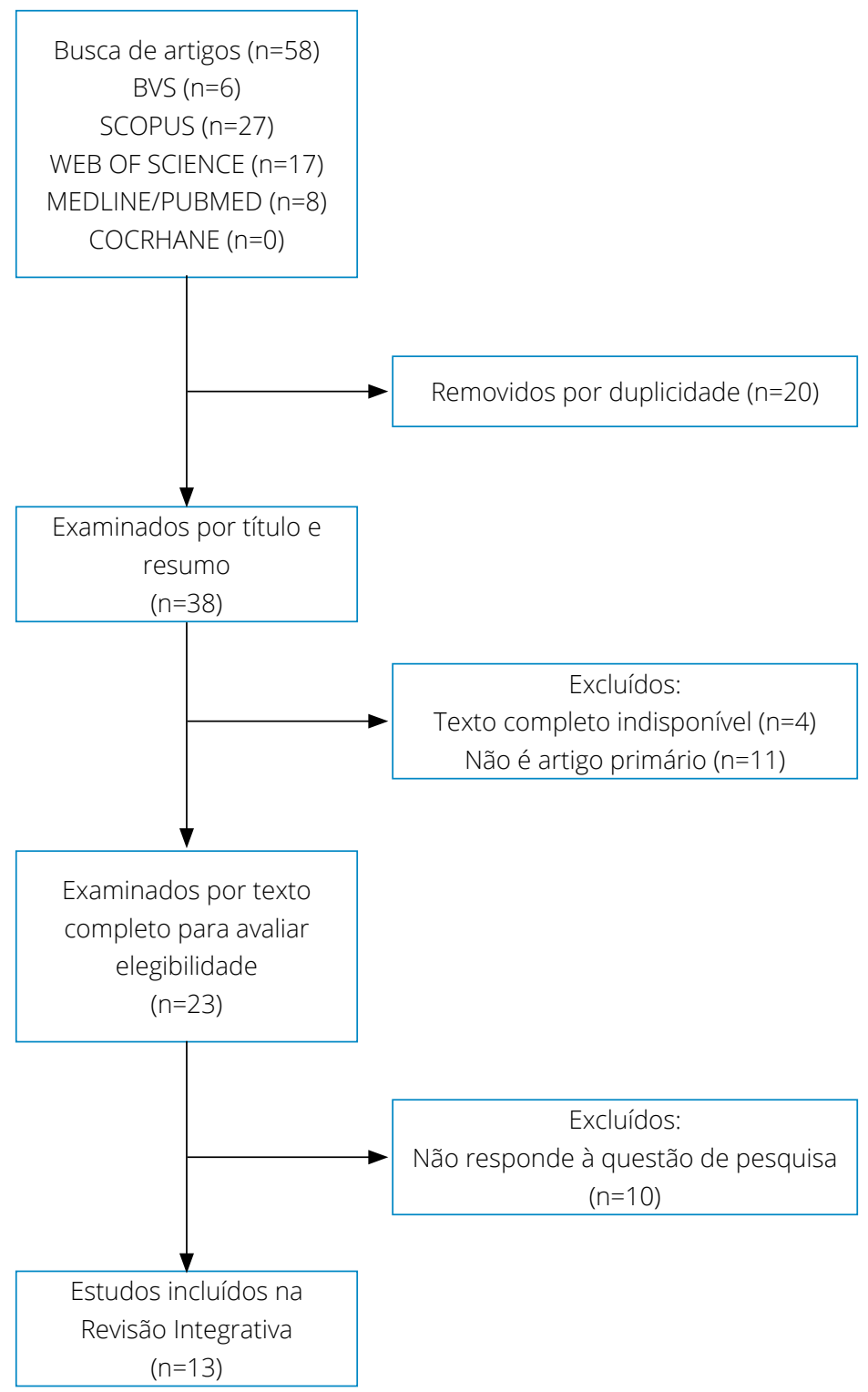

Figura 1. Percurso para recuperação e seleção dos estudos nas bases de dados investigadas. Teresina (PI) - 2019.

\section{RESULTADOS}

Os resultados são expostos na Tabela 2, de acordo com autor principal: ano de publicação, título, local e tipo do estudo, objetivo, intervenção, principais resultados e NE.

O ano com maior quantidade de artigos publicados foi 2002 com 3 estudos, seguido de 2015 com 2 e os demais anos com 1 estudo apenas. Destaca-se que o estudo mais antigo que trabalha com a temática data de 1994 e o mais recente de 2016.

Quanto ao idioma, os 13 artigos foram publicados em inglês. Ressalta-se que os trabalhos mais recentes foram desenvolvidos no Brasil, que também se sobressaiu no quesito localização, sendo representado por 5 pesquisas, seguido dos Estados Unidos da América com 3 estudos. Em relação ao NE, 11 estudos se encontram com classificação IV e 2 com NE II.

O óleo de Melaleuca obteve destaque sobre os demais óleos estudados, por estar presente em seis artigos. Os estudos apresentaram dados de eficácia, associação, comparação, forma de apresentação e formulação. 
Tabela 2. Síntese dos artigos da revisão. Teresina (PI) - 2019.

\begin{tabular}{|c|c|c|c|c|c|c|c|}
\hline $\begin{array}{l}.00 \\
.00 \\
0\end{array}$ & 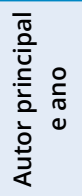 & Título do estudo & $\begin{array}{l}\text { Local do } \\
\text { estudo }\end{array}$ & $\begin{array}{c}\text { Tipo de } \\
\text { estudo e NE }\end{array}$ & Objetivo & $\begin{array}{c}\text { Intervenção } \\
\text { (Óleo } \\
\text { essencial) }\end{array}$ & Principais resultados \\
\hline 飞 & 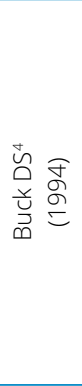 & $\begin{array}{l}\text { Comparação de } \\
\text { duas preparações } \\
\text { tópicas para o } \\
\text { tratamento da } \\
\text { onicomicose: } \\
\text { Melaleuca } \\
\text { alternifolia (tea } \\
\text { tree) e clotrimazol. }\end{array}$ & $\begin{array}{l}\text { Nova York, } \\
\text { Estados } \\
\text { Unidos. }\end{array}$ & $\begin{array}{l}\text { Ensaio clínico } \\
\text { randomizado } \\
\text { controlado; } \\
\text { NE II }\end{array}$ & $\begin{array}{l}\text { Avaliar a eficácia e } \\
\text { tolerabilidade da } \\
\text { aplicação tópica } \\
\text { de } 1 \% \text { de solução } \\
\text { de clotrimazol } \\
\text { comparada com } \\
\text { 100\% de óleo de } \\
\text { Melaleuca alternifolia } \\
\text { (tea tree) para o } \\
\text { tratamento de } \\
\text { onicomicose. }\end{array}$ & $\begin{array}{l}\text { Melaleuca } \\
\text { alternifolia }\end{array}$ & $\begin{array}{l}\text { Todas as terapias atuais têm altas taxas } \\
\text { de recorrência. A terapia oral tem as } \\
\text { desvantagens adicionais de alto custo e } \\
\text { efeitos adversos potencialmente graves. } \\
\text { A terapia tópica, incluindo as duas } \\
\text { preparações apresentadas no estudo, } \\
\text { proporciona melhoria nos sintomas e } \\
\text { na aparência das unhas. O uso de uma } \\
\text { preparação tópica em conjunto com } \\
\text { o desbridamento é uma estratégia de } \\
\text { tratamento inicial adequada. }\end{array}$ \\
\hline$\stackrel{\gtrless}{<}$ & 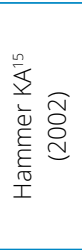 & $\begin{array}{l}\text { Óleo de Melaleuca } \\
\text { alternifolia (tea } \\
\text { tree) inibe a } \\
\text { formação de tubo } \\
\text { germinativo por } \\
\text { Candida albicans. }\end{array}$ & $\begin{array}{l}\text { Nedlands, } \\
\text { Austrália. }\end{array}$ & $\begin{array}{l}\text { Estudo de } \\
\text { coorte; } \\
\text { NE IV }\end{array}$ & $\begin{array}{l}\text { Investigar os efeitos } \\
\text { do óleo de Melelauca } \\
\text { sobre a formação de } \\
\text { tubos germinativos } \\
\text { por Cândida albicans. }\end{array}$ & $\begin{array}{l}\text { Melaleuca } \\
\text { alternifolia }\end{array}$ & $\begin{array}{l}\text { A comparação da suscetibilidade ao TTO de } \\
\text { conídios de Aspergillus niger germinados e } \\
\text { não germinados mostrou que os conídios } \\
\text { germinados são mais suscetíveis do que } \\
\text { os conídios não germinados. Esses dados } \\
\text { demonstram que o óleo da árvore do chá } \\
\text { tem atividade inibitória e fungicida. }\end{array}$ \\
\hline$\underset{z}{m}$ & 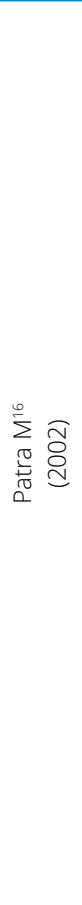 & $\begin{array}{l}\text { Utilização de } \\
\text { óleo essencial } \\
\text { como antifúngico } \\
\text { natural } \\
\text { contra fungos } \\
\text { infecciosos das } \\
\text { unhas. }\end{array}$ & $\begin{array}{l}\text { Allahabad, } \\
\text { Índia. }\end{array}$ & $\begin{array}{l}\text { Estudo de } \\
\text { coorte; } \\
\text { NE IV }\end{array}$ & $\begin{array}{l}\text { Explorar outros } \\
\text { produtos vegetais } \\
\text { (óleos essenciais) } \\
\text { que podem ser } \\
\text { explorados como } \\
\text { fungicidas eficazes. }\end{array}$ & $\begin{array}{l}\text { Foeniculum } \\
\text { vulgare }\end{array}$ & $\begin{array}{l}\text { Durante o rastreio antifúngico de alguns } \\
\text { óleos essenciais, Foeniculum vulgare } \\
\text { apresentou a atividade mais forte, inibindo } \\
\text { completamente o crescimento micelial dos } \\
\text { fungos infecciosos das unhas, Trichophyton } \\
\text { rubrum, T. mentagrophytes e Scytalidium } \\
\text { dimidiatum. O óleo essencial foi encontrado } \\
\text { como fungicida em concentrações de 0,2, } \\
\text { 0,4 e 0,5 } \mu \text { l/ml. O óleo foi eficientemente } \\
\text { ativo contra doses pesadas de inóculo em } \\
\text { concentrações mínimas de fungicidas. A } \\
\text { atividade fungicida do óleo foi encontrada } \\
\text { como termoestável até } 80^{\circ} \text { C, sem } \\
\text { diminuição da atividade descodificável } \\
\text { após } 48 \text { meses de armazenamento. O } \\
\text { óleo também apresentou amplo espectro } \\
\text { fungitóxico, inibindo o crescimento micelial } \\
\text { de outros fungos infecciosos nas unhas, } \\
\text { como Aspergillus flavus, A. fumigatus, A. niger, } \\
\text { A. ustus, Candida albicans, Epidermophyton } \\
\text { floccosum, Microporum audouinii, M. canis, } \\
\text { M. gypseum, M. nanum, Rhizopus nigricans, } \\
\text { Trichophyton tonsurans e T. violaceum. Além } \\
\text { disso, não apresentou quaisquer efeitos } \\
\text { adversos na pele e unhas de mamíferos até } \\
5 \% \text { de concentração. }\end{array}$ \\
\hline 是 & 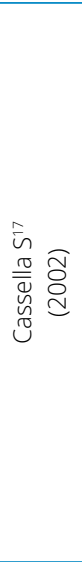 & $\begin{array}{l}\text { Atividade } \\
\text { antifúngica } \\
\text { sinérgica dos } \\
\text { óleos essenciais } \\
\text { da tea tree } \\
\text { (Melaleuca } \\
\text { alternifolia) } \\
\text { e lavanda } \\
\text { (Lavandula } \\
\text { angustifolia) } \\
\text { contra a infecção } \\
\text { dermatofítica. }\end{array}$ & $\begin{array}{l}\text { Derby, Reino } \\
\text { Unido. }\end{array}$ & $\begin{array}{l}\text { Estudo de } \\
\text { coorte; } \\
\text { NE IV }\end{array}$ & $\begin{array}{l}\text { O potencial } \\
\text { antifúngico dos } \\
\text { óleos essenciais de } \\
\text { Melaleuca e Lavanda, } \\
\text { isoladamente e em } \\
\text { combinação, contra } \\
\text { causas comuns de } \\
\text { infecção por tinea } \\
\text { em humanos, foi } \\
\text { investigado através } \\
\text { de investigações } \\
\text { in vitro, a fim de } \\
\text { determinar uma } \\
\text { dosagem adequada } \\
\text { para uso em ensaios } \\
\text { clínicos. }\end{array}$ & $\begin{array}{l}\text { Melaleuca } \\
\text { alternifólia } \\
\text { e Lavandula } \\
\text { angustifolia }\end{array}$ & $\begin{array}{l}\text { Os resultados confirmam que a ação } \\
\text { sinérgica ocorre entre esses dois óleos } \\
\text { essenciais comumente usados na } \\
\text { efetivação da atividade antifúngica. }\end{array}$ \\
\hline
\end{tabular}


Tabela 2. Continuação...

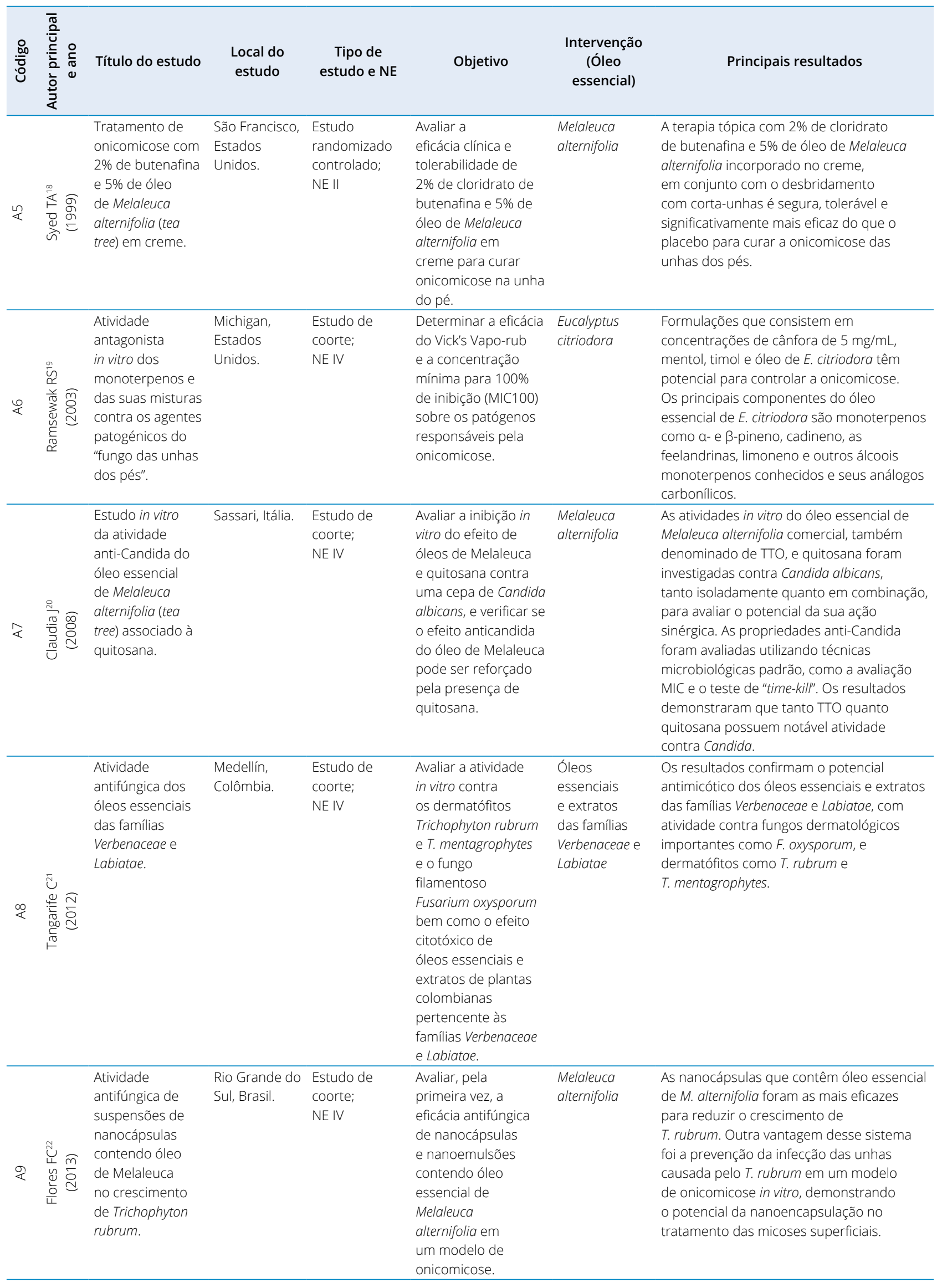


Tabela 2. Continuação...

\begin{tabular}{|c|c|c|c|c|c|c|c|}
\hline $\begin{array}{l}0 \\
\frac{.00}{2} \\
0 \\
0\end{array}$ & 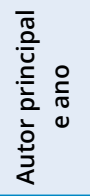 & Título do estudo & $\begin{array}{l}\text { Local do } \\
\text { estudo }\end{array}$ & $\begin{array}{c}\text { Tipo de } \\
\text { estudo e NE }\end{array}$ & Objetivo & $\begin{array}{l}\text { Intervenção } \\
\text { (Óleo } \\
\text { essencial) }\end{array}$ & Principais resultados \\
\hline$\frac{O}{\frac{1}{4}}$ & 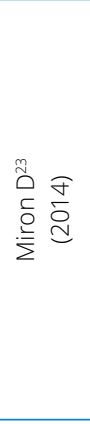 & $\begin{array}{l}\text { Atividade } \\
\text { antifúngica e } \\
\text { mecanismo } \\
\text { de ação dos } \\
\text { monoterpenos } \\
\text { contra } \\
\text { dermatófitos e } \\
\text { leveduras. }\end{array}$ & $\begin{array}{l}\text { Rio Grande do } \\
\text { Sul, Brasil. }\end{array}$ & $\begin{array}{l}\text { Estudo de } \\
\text { coorte; } \\
\text { NE IV }\end{array}$ & $\begin{array}{l}\text { Estabelecer a } \\
\text { atividade antifúngica } \\
\text { de alguns } \\
\text { monoterpenos } \\
\text { encontrados em } \\
\text { óleos voláteis (GOL, } \\
\text { NOL, GAL, NAL e CIT) } \\
\text { e investigar possíveis } \\
\text { mecanismos de } \\
\text { ação para essas } \\
\text { substâncias. }\end{array}$ & Monoterpenos & $\begin{array}{l}\text { A atividade antifúngica de geraniol, nerol, } \\
\text { citral, neral e geranial (monoterpenos) } \\
\text { e terbinafina e anidulafungina (drogas } \\
\text { controle) contra sete leveduras patogênicas } \\
\text { oportunistas e quatro espécies de } \\
\text { dermatófitos foi avaliada pelos testes de } \\
\text { microdiluição. Os monoterpenos foram } \\
\text { mais ativos contra dermatófitos do que } \\
\text { contra as leveduras com GMIC de } 34,5 \text { e } \\
\text { 100,4 } \mu \text { g.ml-1', respectivamente. Ergosterol } \\
\text { relacionando seu mecanismo de ação à } \\
\text { desestabilização da membrana celular. }\end{array}$ \\
\hline 文 & 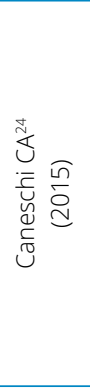 & $\begin{array}{l}\text { Atividade } \\
\text { antifúngica in } \\
\text { vitro do óleo } \\
\text { essencial de } \\
\text { Baccharis trimera } \\
\text { less (DC) contra } \\
\text { dermatófitos. }\end{array}$ & $\begin{array}{l}\text { Rio de Janeiro, } \\
\text { Brasil. }\end{array}$ & $\begin{array}{l}\text { Estudo de } \\
\text { coorte; } \\
\text { NE IV }\end{array}$ & $\begin{array}{l}\text { Identificar } \\
\text { os principais } \\
\text { componentes do } \\
\text { óleo essencial de } \\
\text { Baccharis trimera } \\
\text { Less e investigar sua } \\
\text { atividade antifúngica } \\
\text { in vitro contra sete } \\
\text { cepas fúngicas } \\
\text { causadoras de } \\
\text { onicomicose. }\end{array}$ & $\begin{array}{l}\text { Baccharis } \\
\text { trimera less }\end{array}$ & $\begin{array}{l}\text { O óleo essencial tem atividade antifúngica } \\
\text { contra fungos filamentosos e pode } \\
\text { ser utilizado como alternativa para o } \\
\text { tratamento da onicomicose. O óleo } \\
\text { essencial de } B \text {. trimera tem efeito } \\
\text { antifúngico contra dois dos três gêneros de } \\
\text { fungos filamentosos. }\end{array}$ \\
\hline 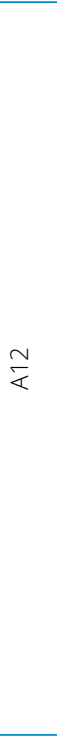 & 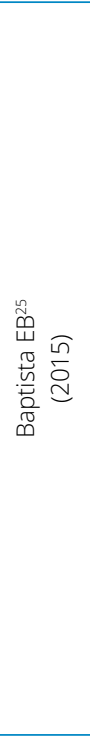 & $\begin{array}{l}\text { Composição } \\
\text { química e } \\
\text { atividade } \\
\text { antifúngica do } \\
\text { óleo essencial } \\
\text { de Eucalyptus } \\
\text { smithii contra } \\
\text { dermatófitos. }\end{array}$ & $\begin{array}{l}\text { Minas Gerais, } \\
\text { Brasil. }\end{array}$ & $\begin{array}{l}\text { Estudo de } \\
\text { coorte; } \\
\text { NE IV }\end{array}$ & $\begin{array}{l}\text { Investigar a atividade } \\
\text { antifúngica do } \\
\text { óleo essencial de } \\
\text { Eucalyptus smithii } \\
\text { R.T. Baker contra os } \\
\text { principais fungos } \\
\text { filamentosos } \\
\text { causadores de } \\
\text { dermatofitose, } \\
\text { como Microsporum } \\
\text { canis, Microsporum } \\
\text { gypseum, Trichophyton } \\
\text { mentagrophytes e } \\
\text { Trichophyton rubrum } \\
\text { in vitro, e determinar } \\
\text { as alterações } \\
\text { micromorfológicas } \\
\text { resultantes desses } \\
\text { fungos, usando } \\
\text { microscopia eletrônica } \\
\text { de varredura (MEV). }\end{array}$ & $\begin{array}{l}\text { Eucalyptus } \\
\text { smithii }\end{array}$ & $\begin{array}{l}\text { Identificou-se o potencial do óleo essencial } \\
\text { de Eucalyptus smithii como agente } \\
\text { terapêutico natural para o tratamento da } \\
\text { dermatofitose. }\end{array}$ \\
\hline$\sum^{m}$ &  & $\begin{array}{l}\text { Análise MDGC- } \\
\text { MS de óleos } \\
\text { essenciais } \\
\text { de Protium } \\
\text { heptaphyllum } \\
\text { (Aubl.) e sua } \\
\text { atividade } \\
\text { antifúngica contra } \\
\text { espécies de } \\
\text { Candida. }\end{array}$ & $\begin{array}{l}\text { Teresina, } \\
\text { Brasil. }\end{array}$ & $\begin{array}{l}\text { Estudo de } \\
\text { coorte; } \\
\text { NE IV }\end{array}$ & $\begin{array}{l}\text { Analisar a composição } \\
\text { química do óleo } \\
\text { essencial da resina } \\
\text { P. heptaphyllum } \\
\text { (OEPh) em diferentes } \\
\text { tempos de extração } \\
\text { e avaliar sua } \\
\text { atividade antifúngica } \\
\text { contra espécies de } \\
\text { Candida isoladas de } \\
\text { horticultores com } \\
\text { onicomicoses, por } \\
\text { método de disco- } \\
\text { difusão. }\end{array}$ & $\begin{array}{l}\text { Protium } \\
\text { heptaphyllum }\end{array}$ & $\begin{array}{l}\text { Constatou-se considerável atividade } \\
\text { antifúngica da resina de óleo de protium, } \\
\text { inibindo espécies de Candida, pelo método } \\
\text { de difusão em disco, com diâmetro de halos } \\
\text { (X>14mm), que causam amplo espectro de } \\
\text { infecções superficiais invasivas e infecções } \\
\text { nas unhas. }\end{array}$ \\
\hline
\end{tabular}

NE = Nível de Evidência; TTO = Óleo da Árvore de Chá; MIC = Concentração Inibitória Mmínima; GOL = Geraniol; NOL = Nerol; GAL = Geranial; NAL = Neral; CIT = Citral; GMIC = Média geométrica da concentração inibitória mínima; MDGC/EM = Cromatografia de gás multidimensional associada à espectrometria de massa. 


\section{DISCUSSÃO}

Os artigos incluídos nessa revisão trazem estudos que buscam comprovar a eficácia antifúngica dos óleos essenciais ${ }^{14,18}$ bem como sua composição e mecanismos de ação $0^{15-17,19-25}$. Incluindo, assim, análises de associação de substâncias, composição, comparação e diferentes formulações contra os principais agentes etiológicos da onicomicose.

$\mathrm{O}$ artigo mais antigo do presente estudo e de maior NE, trata-se de um ensaio clínico duplo-cego, multicêntrico, randomizado e controlado, que aborda a eficácia e tolerabilidade da aplicação tópica de 1\% de solução de clotrimazol (CL) comparada ao óleo puro de Melaleuca alternifolia 100\% (TT) para o tratamento de onicomicose. Encontrando resultados semelhantes com as duas terapias ao final dos 6 meses da pesquisa, com cura da cultura (CL =11\%, TT = 18\%) e resolução parcial ou total $(\mathrm{CL}=61 \%, \mathrm{TT}=60 \%)$ de acordo com avaliação clínica ${ }^{14}$.

O óleo de Melaleuca alternifólia em concentrações diferentes, propostas pelo ensaio clínico, também inibiu reversivelmente a formação de tubos germinativos (FTG) em C. albicans nas maiores concentrações de óleo (0,25\%). No entanto, em concentrações mais baixas (0,125\%), a FTG foi especificamente inibida enquanto o crescimento continuou pela brotação. Esses achados podem ser devidos aos efeitos sobre as membranas celulares e funções associadas, incluindo a inibição da respiração ${ }^{15}$.

Em outros estudos, um de coorte e um clínico randomizado controlado, foram apresentadas associações de componentes ao óleo essencial de Melaleuca alternifólia, buscando conhecimento sobre um suposto efeito fungicida aumentado ${ }^{17-18,}$ ${ }^{20}$. Em um estudo, associaram-se dois óleos essenciais, Melaleuca alternifólia e Lavandula angustifólia, na inibição do Trichophyton rubrum, agente fúngico dermatófito que coloniza a superfície da pele humana e é a principal causa de "pé de atleta" e onicomicose. No dia 7 foi utilizada uma proporção de 30\% v/v lavanda ou 20\% v/v de óleo de Melaleuca resultando em 100\% de inibição. No dia 14, a diluição mínima para manter 100\% de inibição foi de 40\% v/v lavanda ou 30\% v/v do óleo de Melaleuca. Já na análise de sinergia dos óleos, a diluição mínima das misturas de óleos essenciais que dão $100 \%$ de inibição foi de 10\% v/v lavanda com 20\% v/v óleo de Melaleuca ou 20\% v/v lavanda com 10\% v/v óleo de Melaleuca. No dia 14, as culturas que haviam sido expostas a essas misturas também mantiveram 100\% de inibição. Confirmando, assim, que a ação sinérgica ocorre entre esses dois óleos essenciais comumente usados na efetivação da atividade antifúngica ${ }^{17}$.

Outra associação testada em estudo clínico foi a terapia tópica com 5\% de óleo de Melaleuca alternifolia e 2\% de cloridrato de butenafina. Esse creme, em conjunto com o desbridamento com um corta-unhas, foi considerado seguro, tolerável e significativamente mais eficaz do que o placebo (somente óleo de Melaleuca) para curar a onicomicose das unhas dos pés. Resultado evidenciado após constatar $80 \%$ de cura no grupo que utilizou a associação por 8 semanas, contra nenhum caso de cura no grupo que utilizou o placebo. Levantando a hipótese de que o tempo não tenha sido suficiente para cura no grupo que utilizou apenas o óleo de Melaleuca, visto que esse possui eficácia comprovada por vários estudos ${ }^{18}$.

$\mathrm{Na}$ avaliação do efeito antifúngico, em estudo de coorte, contra Candida albicans do óleo de Melaleuca associado à quitosana, foi constatado que essa não melhorou significantemente a atividade do óleo, achado que pode ser explicado devido ao tempo maior que as concentrações inibitórias de quitosana necessitam para mostrar sua atividade. Individualmente o óleo de Melaleuca inibiu C. albicans a 0,5\% v/v e foi capaz de matar células de leveduras em 120 minutos a 1\% e em 300 minutos entre 0,5 e $0,25 \%$. Em relação à quitosana, foram necessários $0,5 \mathrm{mg} / \mathrm{mL}$ para inibir o crescimento de Candida em meio líquido, enquanto $0,5-2 \mathrm{mg} / \mathrm{mL}$ de quitosana foram necessários 24 horas para matá-los no teste de avaliação da cinética de morte bacteriana $(\text { time-kill })^{20}$.

O efeito terapêutico do óleo de Foeniculum vulgare foi investigado através de um estudo de coorte que o considerou fungicida nas concentrações de 0,2; 0,4 e 0,5 $\mu \mathrm{l} / \mathrm{ml}$, nos tempos de 50,60 e 80 minutos, contra Trichophyton rubrum, T. mentagrophytes e Scytalidium dimidiatum, respectivamente. Já o óleo puro (100\%) matou os fungos em apenas 5 segundos. Além dos agentes citados anteriormente, o óleo também se mostrou eficaz contra Candida albicans, Epidermophyton floccosum, M. canis, entre outros, na faixa de concentração de 0,5-1,0 $\mu 1 / \mathrm{ml}$. Entre suas características, pode-se citar a longa vida de prateleira, termoestabilidade e ausência de efeitos adversos ${ }^{16}$. 
A eficácia do Vick's Vapo-rub e a concentração mínima para 100\% de inibição sobre os patógenos responsáveis pela onicomicose foram determinadas por meio de um estudo de coorte in vitro. Os dados de inibição revelaram que cânfora, mentol, timol e o óleo de Eucalyptus citriodora foram os componentes mais eficazes contra os organismos em teste (Tricophyton rubrum, Trichophyton mentagrophytes, Microsporum canis, Epidermophyton floccosum e Epidermophyton stockdale). A concentração mínima para inibição de 100\% para misturas desses quatro componentes em vários solventes revelou que as formulações com presença de concentrações de $5 \mathrm{mg} / \mathrm{mL}$ de cada uma tem potencial para ser eficaz no controle da onicomicose ${ }^{19}$.

A atividade antifúngica de 14 óleos essenciais (Aloysia triphylla, Lippia alba N.E.Brown, Lippia micrômera Schauer, Lippia origanoides Kunth, Lantana fucata Lindl) e 1 extrato (Lantana fucata Lindl) pertencentes às famílias Verbenaceae, e 3 óleos (Minthostachys mollis Griseb, Origanum vulgare) e 2 extratos (Lepechinia conferta e Salvia melaleuca subsp. Melaleuca) pertencentes a família Labiatae foi forte entre $70 \%$ e $80 \%$ das amostras avaliadas contra dermatófitos (Trichophyton rubrum e T. mentagrophytesde) e $20 \%$ contra F. oxysporum. Ressalta-se que a mesma planta teve seu óleo extraído em diferentes regiões de plantio e épocas do ano, permitindo mais opções de análise como detalhado no estudo. Os menores valores de Concentração Inibitória Mínima (MIC) foram obtidos com o quimiotipo citral óleo de Lippia alba nas concentrações de 31,25 e $125 \mu \mathrm{g} / \mathrm{mL}$ em T. rubrum e T. mentagrophytes, respectivamente, mas não em F. oxysporum ${ }^{21}$.

Além disso, o óleo de Minthostachys mollis Griseb apresentou forte atividade contra todos os fungos avaliados. Os óleos essenciais com os maiores valores de índice de seletividade foram Aloysia triphylla e óleo de L. alba nos dermatófitos. O principal componente dos óleos mais ativos de L. alba foi caracterizado por carvona e citral. Para L. origanoides foram encontrados o carvacrol e o timol como componente principal. Pulegona e epóxido de cis-piperitona foram os principais constituintes dos óleos Minthostachys mollis. A presença desses componentes principais nos óleos essenciais pode ser responsável pela atividade antifúngica. Esses achados são muito importantes porque confirmam o potencial dos óleos essenciais como fonte de novos antidermatófitos ${ }^{21}$.

A atividade antifúngica de suspensões de nanocápsulas contendo óleo de Melaleuca foi avaliada, por estudo de coorte, no crescimento de Trichophyton rubrum, um dos principais causadores de onicomicose. Os resultados evidenciaram uma maior eficácia no uso de nanocápsulas comparadas com nanoemulsão e emulsão convencional. Nanocápsulas, ao contrário das nanoemulsões, são formados por uma parede polimérica disposta ao redor do núcleo de óleo, que protege a entrega imediata do óleo, fornecendo, por hipótese, uma liberação gradual de óleo e mantendo suas concentrações por mais tempo ${ }^{22}$. Estudo anterior realizado pela mesma autora também traz que a inclusão do óleo de Melaleuca nas nanocápsulas mostrou maior proteção contra a volatilização ${ }^{27}$.

Em estudo de coorte realizado no Brasil foram apresentados resultados positivos dos monoterpenos, presentes nos óleos essenciais, no "Teste de afinidade com Ergosterol" e os outros relatos sobre o assunto sugerem fortemente que o mecanismo de ação dessa classe de medicamentos está relacionado à ligação ergosterol e à subsequente desestabilização das membranas celulares fúngicas ${ }^{23}$.

O óleo essencial de B. trimera (Concentração Fúngica Mínima/MFC $=0,06 \mu \mathrm{g} \mathrm{mL}^{-1}$ ) foi mais potente do que os medicamentos terbinafina e cetoconazol ( $\mathrm{MFC}=0,19$ e 4,00 $\mu \mathrm{g} \mathrm{mL} \mathrm{m}^{-1}$, respectivamente) contra $T$. rubrum e apresentou menor valor de $\operatorname{MFC}\left(0,98 \mu \mathrm{g} \mathrm{mL}^{-1}\right)$ que cetoconazol $\left(8,01 \mu \mathrm{g} \mathrm{mL}{ }^{-1}\right)$ contra $M$. canis. Em outras palavras, a atividade antifúngica do óleo essencial contra a duas cepas de fungos, T. rubrum e $M$. canis, foi maior quando comparada com a de cetoconazol ${ }^{24}$. Esses dados estão de acordo com outros estudos que afirmaram que plantas do gênero Baccharis L. são usados na Argentina e outros países da América do Sul para o tratamento de doenças fúngicas ${ }^{28}$.

O composto 1,8-cineol é o componente predominante (72,2\%) do óleo essencial de Eucalyptus smithii. Os valores de MIC do óleo variaram de $62,5 \mu \mathrm{g} \cdot \mathrm{mL}^{-1} \mathrm{a}>1.000 \mu \mathrm{g} \cdot \mathrm{mL}^{-1}$, e os valores de MFC do óleo variaram de $125 \mu \mathrm{g} \cdot \mathrm{mL}^{-1}$ a > $1.000 \mu \mathrm{g} \cdot \mathrm{mL}^{-1}$. A análise por varredura microscópica eletrônica mostrou dano físico e morfológico como alterações nos fungos expostos a esse óleo, demostrando o potencial do óleo essencial de Eucalyptus smithii como agente terapêutico natural para o tratamento da dermatofitose ${ }^{25}$. 
Contra espécies de Candida isoladas de horticultores com onicomicoses, por método de disco-difusão, o óleo essencial da resina Protium heptaphyllum apresenta atividade antifúngica. Resultado importante se tratando de uma das principais espécies causadoras da onicomicose. Demonstrando inibição, inclusive, com C. krusei, naturalmente resistente ao fármaco fluconazol, situação semelhante ocorreu com a cepa de C. parapsilosis ${ }^{26}$.

Os resultados dos estudos sugerem evidências de que os óleos essenciais se mostram eficazes e benéficos no tratamento para onicomicose. Sendo assim, os profissionais de saúde envolvidos nos cuidados com paciente com onicomicose devem se manter atualizados sobre tais alternativas para o tratamento da onicomicose e consequentemente da melhora da qualidade de vida desses pacientes.

Como limitação para elaboração dessa revisão integrativa, destaca-se a presença de estudos com baixa evidência científica, assim como poucos estudos clínicos na temática discutida.

\section{CONCLUSÃO}

Diante dos artigos analisados, pode-se afirmar que os estudos sobre óleos essenciais apresentam dados promissores, porém esses são em sua maioria provenientes de estudos pré-clínicos, ainda que nos dois ensaios clínicos incluídos os dados tenham apontado para eficácia terapêutica.

Os estudos clínicos apresentados apontam que os óleos essenciais, quando comparados a outras terapias, mostraram-se tão eficazes quanto as terapias convencionais, como clotrimazol, terbinafina e cetoconazol.

Entretanto, a maior parte dos estudos identificados possui baixo nível de evidência por serem estudos pré-clínicos, mostrando, assim, a necessidade da produção de estudos com delineamento experimental, que possam fundamentar a aplicação na prática clínica com segurança, visando, também, à contribuição nos cuidados de pacientes acometidos por essa patologia.

\section{CONTRIBUIÇÃO DOS AUTORES}

Conceitualização: Ribeiro TB, Bezerra SMG, Lira JAC e Oliveira RA; Metodologia: Ribeiro TB, Lira JAC, Machado RS, Bezerra SMG e Oliveira RA; Investigação: Ribeiro TB, Bezerra SMG, Lira JAC e Oliveira RA; Redação - Primeira versão: Ribeiro TB, Bezerra SMG, Lira JAC, Oliveira RA e Machado RS; Redação - Revisão \& Edição: Ribeiro TB; Lira JAC; Bezerra SMG; Oliveira RA; Machado RS e Nogueira LT; Supervisão: Ribeiro TB.

\section{DISPONIBILIDADE DE DADOS DE PESQUISA}

Não se aplica

\section{REFERÊNCIAS}

1. Vlahovic TC. Onychomycosis: Evaluation, Treatment Options, Managing Recurrence, and Patient Outcomes. Clin Podiatr Med Surg. 2016;33(3):305-18. https://doi.org/10.1016/j.cpm.2016.02.001

2. Elewski BE, Tosti A. Risk Factors and Comorbidities for Onychomycosis: Implications for Treatment with Topical Therapy. J Clin Aesthet Dermatol. 2015;8(11):38-42. Disponível em: https://www.ncbi.nlm.nih.gov/pmc/articles/PMC4689496/pdf/ jcad_8_11_38.pdf

3. Maquiné GA. Estudo descritivo dos casos de onicomicoses atendidos em um centro de referência em dermatologia na cidade de Manaus [dissertação]. Manaus (AM): Mestrado em doenças tropicais e infecciosas, Universidade do Estado do Amazonas; 2017.

4. Maranhão FCA, Oliveira-Júnior JB, Araújo, MAS, Silva DMW. Mycoses in northeastern Brazil: epidemiology and prevalence of fungal species in 8 years of retrospective analysis in Alagoas. Braz J Microbiol. 2019;50(4):969-78. https://doi.org/10.1007/ s42770-019-00096-0 
5. Rato M, Costin A, Furtado C, Sousa C, Toscano C, Veríssimo C et al. Epidemiologia das Infeções Fúngicas Superficiais em Portugal: Revisão de 3 Anos (2014-2016). Revista SPDV 2018;76(3):269-78. https://doi.org/10.29021/spdv.76.3.910

6. Achterman RR, White TC. A foot in the door for dermatophyte research. PLoS Pathog. 2012;8(3):e1002564. https://doi. org/10.1371/journal.ppat.1002564

7. Morales-Cardona CA, Valbuena-Mesa MC, Alvarado Z, Solorzano-Amador A. Non-dermatophyte mould onychomycosis: a clinical and epidemiological study at a dermatology referral centre in Bogota, Colombia. Mycosis. 2014;57(5):284-93. http://doi. org/10.1111/myc.12157

8. Andrei P, Comune AP. Aromaterapia e suas aplicações. Centro Universitário S. Camilo 2005;11(4):57-68. Disponível em: http:// www.saocamilo-sp.br/pdf/cadernos/36/07_aromaterapia.pdf

9. Cóbar MLP. Aromaterapia [dissertação]. Guatemala. Dissertação: Facultad de Humanidades, Universidad de San Carlos de Guatemala; 2005.

10. Bizzo HR, Hovell AMC, Rezende CM. Óleos essenciais no Brasil: aspectos gerais, desenvolvimento e perspectivas. Quim Nova 2009;32(3):588-94. https://doi.org/10.1590/S0100-40422009000300005

11. Serafini LA, Santos ACA, Touguinha LA, Agostini G, Dalfovo V. Extrações e aplicações de óleos essenciais de plantas aromáticas e medicinais. $1^{\text {a }}$ ed. Caxias do Sul: Educs; 2002.

12. Mendes KDS, Silveira RCCP, Galvão CM. Revisão integrativa: método de pesquisa para a incorporação de evidências na saúde e na enfermagem. Texto Contexto Enferm. 2008;17(4):758-64. Disponível em: http://www.scielo.br/pdf/tce/v17n4/18.pdf

13. Melnyk BM, Fineout-Overholt E. Evidence-based practice in nursing \& healthcare: a guide to best practice. $3^{\mathrm{a}}$ ed. Philadelphia: Lippincott Williams \& Wilkins; 2014.

14. Buck DS, Nidorf DM, Addino JG. Comparison of two topical preparations for the treatment of onychomycosis: Melaleuca alternifolia (tea tree) oil and clotrimazole. J Fam Pract. 1994;38(6):601-5. Disponível em: https://pubmed.ncbi.nlm.nih. gov/8195735/

15. Hammer KA, Carson CF, Riley TV. In vitro activity of Melaleuca alternifolia (tea tree) oil against dermatophytes and other filamentous fungi. J Antimicrobial Chemother. 2002;50(2):195-9. https://doi.org/10.1093/jac/dkf112

16. Patra M, Shahi SK, Midgely G, Dikshit A. Utilization of essential oil as natural antifungal against nail-infective fungi. Flavour Fragr J. 2002;17(2):91-4. https://doi.org/10.1002/ffj.1049

17. Cassella S, Cassella JP, Smith I. Synergistic antifugal activity of tea tree (Melaleuca Alternifolia) and lavender (Lavandula angustifolia) essential oils against dermatophyte intection. Int J Aromath. 2002; 12(1):2-15. https://doi.org/10.1054/ ijar.2001.0127

18. Syed TA, Qureshi ZA, Ali SM, Ahmad S, Ahmad SA. Treatment of toenail onychomycosis with $2 \%$ butenafine and $5 \%$ Melaleuca alternifólia (tea tree) oil in cream. Trop Med Int Health. 1999;4(4):284-7. https://doi.org/10.1046/j.1365-3156.1999.00396.x

19. Ramsewak RS, Nair MG, Stommel M, Selanders L. In vitro antagonistic activity of monoterpenes and their mixtures against 'toe nail fungus' pathogens. Phytother Res. 2003;17(4):376-9. https://doi.org/10.1002/ptr.1164

20. Juliano C, Demurtas C, Piu L. In vitro study on the anticandidal activity of Melaleuca alternifolia (tea tree) essential oil combined with chitosan. Flavour Fragr J. 2008;23(4):227-31. https://doi.org/10.1002/ffj.1871

21. Castaño VT, Linares VR, Betancur L, Durán DC, Stashenko E, Mesa AC. Antifungal activity of Verbenaceae and Labiatae families essential oils. PharmacologyOnline. 2012; 1(special issue):133-45. Disponível em: https://www.researchgate.net/ profile/Liliana-Betancur/publication/256475420_Antifungal_activity_of_Verbenaceae_and_Labiatae_families_essential_oils/ links/00b49523662d20ec55000000/Antifungal-activity-of-Verbenaceae-and-Labiatae-families-essential-oils.pdf

22. Flores FC, Lima JA, Ribeiro RF, Alves SH, Rolim CMB, Beck RCR et al. Antifungal Activity of Nanocapsule Suspensions Containing Tea Tree Oil on the Growth of Trichophyton rubrum. Mycopathologia. 2013;175(3-4):281-6. https://doi.org/10.1007/s11046013-9622-7

23. Miron D, Battisti F, Silva FK, Lana AD, Pippi B, Casanova B et al. Antifungal activity and mechanism of action of monoterpenes against dermatophytes and yeasts. Rev bras farmacogn. 2014;24(6):660-7. https://doi.org/10.1016/j.bjp.2014.10.014

24. Caneschi CA, Martins FJ, Larrudé DG, Romani EC, Brandão MAF, Raposo NRB. In vitro Antifungal Activity of Baccharis trimera Less (DC) Essential Oil against Dermatophytes. Trop J Pharm Res. 2015;14(11):2083-9. https://doi.org/10.4314/tjpr.v14i11.19

25. Baptista EB, Zimmermann-Franco DC, Lataliza AAB, Raposo NRB. Chemical composition and antifungal activity of essential oil from Eucalyptus smithii against dermatophytes. Rev Soc Bras Med Trop. 2015;48(6):746-52. https://doi.org/10.1590/00378682-0188-2015

26. Mobin M, Lima SG, Almeida LTG, Takahashi JP, Teles JB, Szeszs MW et al. MDGC-MS analysis of essential oils from Protium heptaphyllum (Aubl.) and their antifungal activity against Candida specie. Rev bras plantas med. 2016;18(2):531-8. https://doi. org/10.1590/1983-084X/15_110 
27. Flores FC. Sistemas nanoestruturados contendo óleo essencial de Melaleuca alternifólia: desenvolvimento de formulações e atividade biológica [dissertação]. Santa Maria (RS): Mestrado do Programa de Pós-graduação em ciências farmacêuticas, Universidade Federal de Santa Maria; 2011.

28. Rodriguez MV, Sortino MA, Ivancovich JJ, Pellegrino JM, Favier LS, Raimondi MP et al. Detection of synergistic combinations of Baccharis extracts with Terbinafine against Trichophyton rubrum with high throughput screening synergy assay (HTSS) followed by 3D graphs. Behavior of some of their componentes. Phytomedicine. 2013;20(13):1230-9. https://doi.org/10.1016/j. phymed.2013.06.015 